\title{
Seroprevalence of hepatitis a virus antibody in overseas workers
}

\begin{abstract}
Background: HAV infection is still a common infectious disease, and can easily spread by the fecal-oral route, either via person-to-person contact or consumption of contaminated food or water. People at high risk of HAV infection include overseas travelers and workers from high endemic areas. HAV seroprevalence rate of international traveled healthy worker was not reported in Taiwan.
\end{abstract}

Aim: Survey the prevalence of seroprevalence of hepatitis A antibody in overseas workers.

Method: A total of 406 healthy overseas workers of three international companies during their annual health examinations were enrolled and examined for anti-hepatitis a virus $\mathrm{IgG}$.

Result: Of these 406 participants, 158(38.9\%) were found to be anti-hepatitis A virus $\mathrm{IgG}$ positive. The sero-positive rate of anti-HAV was significantly different between overseas workers aged below or equal to 30 years and those above 30 years $(0 \%$ vs. $45.9 \%, \mathrm{p}<0.0001)$.

Conclusion: The vast majority of overseas workers aged $\leq 30$ years are susceptible to HAV infection. Mass hepatitis A vaccination should be applied to overseas workers $\leq 30$ years or that sero-negative for anti-HAV after screening.

Keywords: hepatitis a, overseas workers, seroprevalence, organisation for economic co-operation and development, Taiwanese
Volume 3 Issue 3 - 2017

\author{
Yang Che Kuo,' Kuang Chun Hu, ${ }^{1,2,3}$ Shou \\ Chuan Shih ${ }^{1,3}$ \\ 'Department of Internal Medicine, Division of Gastroenterology, \\ Mackay Memorial hospital, Taiwan \\ ${ }^{2}$ National Taiwan University College of Medicine, Taiwan \\ ${ }^{3}$ Department of Medicine, Nursing and Management College, \\ Taiwan
}

Correspondence: Yang Che Kuo, Department of Internal Medicine, Division of Gastroenterology, Mackay Memorial Hospital, No.92, Sec. 2, Chung Shan North Road, Taipei, Taiwan, Tel 88622543 3535, Email kuoyangche@gmail.com

Received: May 21, 2017 | Published: July 31, 2017

\section{Introduction}

Hepatitis A (HAV) infection is still a common infectious disease, and usually spread by the fecal-oral route, either via person-to-person contact or consumption of contaminated food or water. The main transmission modes of HAV include person-to-person contact or food-borne infection. ${ }^{1}$ The severity of hepatitis A increases with age, usually asymptomatic in children but may cause clinically apparent disease in adults. Although most hepatitis A cases can complete recover from acute hepatitis without sequela, the mortality rate can reach $1.8 \%$ in patients over 50years of age. ${ }^{2}$ People at high risk of HAV infection include overseas travelers and workers from high endemic areas. World financial crisis in 2008 and 2009 has changed the map of global migration however; the Organisation for Economic Co-operation and Development (OECD) claimed that migration into its member countries dropped by $7 \%$ in 2009 . This reduction may be reasoned by the emergence of 'new international division of labour', in which the production processes were relocated from developed countries to developing countries in Asia. ${ }^{3}$ The risk of hepatitis A infection for people traveling abroad varies with living conditions, length of stay, and the incidence of HAV infection in the area where they visit. ${ }^{3}$ Seroprevalence rate of anti-HAV in overseas workers has not been reported yet in Taiwan as to our knowledge. In addition, there is no official recommendation regarding hepatitis A vaccination for overseas workers. We thus studied the seroprevalence rate of antiHAV in a group of overseas workers in Taiwan from 2009 to 2010.

\section{Methods}

This retrospective study included three international companies during their annual health examinations for Taiwanese overseas workers. All of the 415 workers were included, 9 who had received hepatitis A vaccination before was excluded. Finally, a total of 406 overseas workers were enrolled. The presence of immunoglobulin anti-bodies to HAV (HAV IgG) was tested by using a commercial electrochemiluminescence immunoassay kit (COBAS E 170, Roche Diagnostics, Indiana polis, In, USA). The association of grades with seroprevalence was tested using the Chi-square $\left(\chi^{2}\right)$ test and the relationship between age groups and seroprevalence was analyzed using the $\chi^{2}$ test for trend. Statistical significance was set at $\mathrm{P}<0.05$. This data of study was delinked for the protection of subject's privacy, and reviewed and approved by the Ethical Committee of Mackay Memorial Hospital.

\section{Results}

Of 406 participants [358 males and 48 females, mean age: 40.3years (range: 22-73)], the overall sero-positive rate of antiHAV IgG was $38.9 \%$ (158 subject), implying $61.1 \%$ of Taiwanese overseas workers were not immune to HAV infection. Sero-positivity of HAV is significantly higher in males and increased with age, $0 \%$ for $21-25$ years and $26-30$ years, $11.2 \%$ for $31-35$ years, $27.9 \%$ for $36-40$ years, $50 \%$ for $41-45$ years, $73.3 \%$ for $46-50$ years, $86.1 \%$ for $51-55$ years, $87.6 \%$ for $56-60$ years and $90 \%$ for $>60$ years (Table 1 ). 
The sero-positive rate of anti-HAV was significantly different between overseas workers aged below 30years and those above 30years $(0 \%$ vs. $45.9 \%, \mathrm{p}<0.0001)$. Therefore, age was an independent factor for HAV prior exposure.

Table I Seroprevalence Rates of Anti-Hepatitis a Virus among Different Subsets of Study Population

\begin{tabular}{lllll}
\hline Characteristics & Participants & Sero-positives & Rate & P value \\
\hline All & 406 & 158 & $38.90 \%$ & \\
Gender & & & & \\
Women & 48 & 27 & $56.30 \%$ & 0.005 \\
Men & 358 & 131 & $36.60 \%$ & \\
Age & & & & \\
$\leq 30$ & 62 & 0 & $0 \%$ & \\
$31-35$ & 107 & 12 & $11.20 \%$ & \\
$36-40$ & 68 & 19 & $27.90 \%$ & \\
$41-45$ & 32 & 16 & $50.00 \%$ & $<0.001$ \\
$46-50$ & 60 & 44 & $73.30 \%$ & \\
$51-55$ & 43 & 37 & $86.10 \%$ & \\
$56-60$ & 24 & 21 & $87.50 \%$ & \\
$>60$ & 10 & 9 & $90.00 \%$ & \\
\hline
\end{tabular}

\section{Discussion}

People at high risk for HAV infection include international travelers to high-risk areas, persons residing in day care centers, men who have sex with men, users of injected and non-injected drugs, persons with clotting factor disorders, and persons working with primates. ${ }^{4}$ The risk of HAV infection for international travelers included country of origin, travel destination, duration of stay and personal habits during travel. Different incidence rates in different parts of the developing countries than in developed countries. However, HAV infection is a vaccine preventable disease in international travelers. ${ }^{5}$ From Economist report, after financial crisis the economic situation had changed and more jobs opportunity had shift from western to eastern world. Asia and China are becoming destinations of choice for many young workers. According to Chinese statistics, last year Shanghai had 143,000 foreigners with resident's visas. That does not count thousands of Westerners believed to be there on tourism visas, or the illegals from elsewhere in Asia. ${ }^{6}$ In our study, most Taiwanese overseas workers worked in China. Although the annual incidence of hepatitis A in China has declined by $90 \%$ since 1990 , from 56 to 5.9 per $10^{5} /$ year, western people are still at risk of exposure to HAV because the epidemiology of hepatitis A transmission in China remains unclear. ${ }^{7}$ In Taiwan, the seroprevalence rate of hepatitis A became much lower in recent years, especially in younger people. Previous studies showed the seroprevalence generally increased with age. ${ }^{8-9}$ Wang et al. ${ }^{8}$ reported the seroprevalence rate of anti-HAV in young people (20 to 30years) was $18.2 \%$ in $1998 .{ }^{8} \mathrm{Su}$ et al. ${ }^{9}$ also reported the sero-positivity of HAV was $11.4 \%$ for subjects aged $<30$ years in $2005 .{ }^{9}$ In this study, our data showed that the seroprevalence rate of HAV was $0 \%$ in overseas workers younger than 30years. This discrepancy may be explained by the use of disposable tableware since 1982 this measure is proven as an effective way to reduce hepatitis A transmission. Other factors such as improvement in environmental sanitation might also contribute to the reduction in the seroprevalence of hepatitis A. ${ }^{9}$ It is known that the most common risk factor for hepatitis $\mathrm{A}$ is international travel ${ }^{10}$ and the effectiveness of hepatitis A vaccination is close to $100 \%$. However, only 9 participants (2.2\%) received HAV vaccination in this study, implying the coverage of hepatitis A vaccinated in Taiwan is far from satisfactory. Since most of our study participants are now working in China, and some of them may change the job location to India or Brazil in the future when global economic status changes. Considering the age and current seroprevalence rate of anti-HAV in these overseas workers, they are still at risk of contracting HAV and hepatitis A vaccination should be advised.

In conclusion, the vast majority of Taiwanese overseas workers younger than 30years are susceptible to HAV infection. On the basis of our findings, hepatitis A vaccination should be applied to overseas workers sero-negative for anti-HAV.

\section{Acknowledgements}

None.

\section{Conflict of interest}

Author declares that there is no conflict of interest.

\section{References}

1. Chodick G, Ashkenazi S, Lerman Y. The risk of hepatitis A infection among healthcare workers: a review of reported outbreaks and seroepidemiologic studies. J Hosp Infect. 2006;62:414-420.

2. Lavanchy D. Hepatitis, viral. In Control of communicable diseases manual. In: Heymann DL, editors. American Public Health Association, Washington, USA; 2004. p. 247-253.

3. Koser K. The Global Financial Crisis and International Migration: Policy Implications for Australia. Australia: Lowy Institute for International Policy; 2009.

4. Fiore AE, Wasley A, Bell BP. Prevention of hepatitis A through active or passive immunization. MMWR Recomm Rep. 2006;55(RR07):1-23.

5. Steffen R. Despite good tools, we do not effectively reduce traveler's hepatitis A. J Travel Med. 2002;9(1):1-2.

6. The Economist: Migration after the crash: Moving out, on and back, UK; 2011.

7. Cui F, Hadler SC, Zheng H, et al. Surveillance and vaccine use in China from 1990 through 2007. J Epidemiol. s2009;19(4):189-195.

8. Wang SM, Liu CC, Huang YS, et al. Change in hepatitis A virus seroepidemiology in southern Taiwan: a large percentage of the population lack protective antibody. J Med Virol. 2001;64(2):104-108.

9. Su SB, Lin CY, Sheu MJ, et al. Decrease in seroprevalence of hepatitis A after the implementation of nationwide disposable tableware use in Taiwan. BMC Public Health. 2010;10:719-726.

10. Daniels D, Grytdal S, Wasley A. Surveillance for acute viral hepatitisUnited States, 2007. MMWR Surveill Summ. 2009;58(3):1-27. 